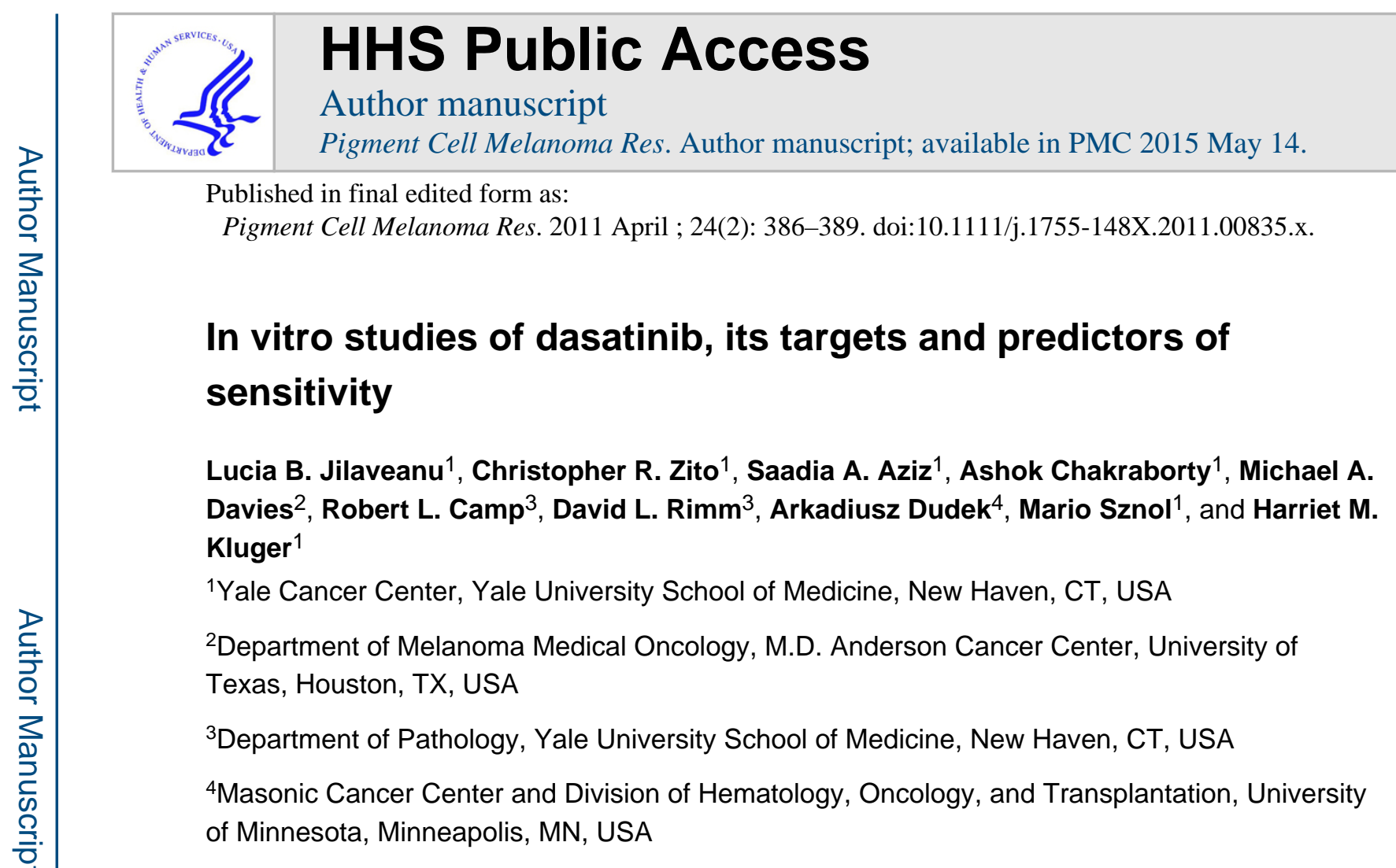

\title{
Dear Editor
}

Src family kinases (SFKs) regulate important cellular events including cell growth, survival, differentiation, adhesion, motility, migration, and invasion. Src family kinases include nine non-receptor membrane-associated proteins: c-Src, c-Yes, Fyn, Lyn, Hck, Lck, Blk, Fgr, and Yrk. Elevated levels and kinase activity of c-Src were reported in melanoma skin metastases compared to normal skin and in melanoma cell lines compared to a nevus cell line (Barnekow et al., 1987). Higher c-Yes was seen in 20 melanoma cell lines compared to melanocytes (Loganzo et al., 1993). Fyn regulates melanoma cell motility and metastatic potential of melanoma cells in mice and controls malignant melanocyte transformation (Huang et al., 2003; Wellbrock and Schartl, 2000).

Dasatinib (BMS-354825), a tyrosine kinase inhibitor, targets BCR-ABL, SFKs, c-KIT, $\mathrm{PDGF} \beta$ receptor, and EPHA2. Dasatinib is the only SFK inhibitor approved in the USA and Europe for leukemias and gastrointestinal stromal tumors. Trials of dasatinib in other tumors are ongoing.

Given the need for new, effective therapies for metastatic melanoma, and because of evidence of Src pathway involvement in melanoma progression, we studied the expression of Src pathway members in human melanomas and investigated Src inhibition and predictors of sensitivity to dasatinib. Expression of c-Src, c-Yes, and Fyn was studied on

() 2011 John Wiley \& Sons A/S

Correspondence Harriet M. Kluger, Harriet.Kluger@yale.edu.

Supporting information

Additional Supporting Information may be found in the online version of this article:

Please note: Wiley-Blackwell are not responsible for the content or functionality of any supporting materials supplied by the authors.

Any queries (other than missing material) should be directed to the corresponding author for the article. 
large cohort tissue microarrays (TMAs) using a method of automated, quantitative analysis (AQUA) (Jilaveanu et al., 2009; Figure S1, Table S1).

By Spearman's correlation test, we found strong co-expression of the three Src kinases ( $\rho=$ $0.488, \mathrm{P}<0.0001$ for c-Src and c-Yes, $\rho=0.658, \mathrm{P}<0.0001$ for c-Src and Fyn and $\rho=$ 0.522 , $\mathrm{P}<0.0001$ for $\mathrm{c}-$ Yes and Fyn). Expression of the SFKs was higher in malignant versus benign tissues ( $\mathrm{P}<0.0001$ for all), Figure 1(A-C). c-Src and Fyn expression was strongly associated with disease stage; expression was lower in primary than metastatic specimens ( $t$-statistic $=4.562, \mathrm{P}<0.0001$ for $\mathrm{c}$-Src; $t$-statistic $=4.577, \mathrm{P}<0.0001$ for Fyn). High c-Src, c-Yes, and Fyn expressions were correlated with high Clark level (IV/V) $(\mathrm{P}=$ $0.0008, \mathrm{P}=0.0067$ and $\mathrm{P}=0.0093$, respectively) but not with other clinical variables.

By Cox univariate analysis, high c-Src expression was correlated with decreased survival in primary specimens $(\mathrm{P}=0.0364)$. On multivariate analysis $\mathrm{c}-\mathrm{Src}$ expression did not retain its independent prognostic value. To visually demonstrate the associations between c-Src and survival, we dichotomized c-Src AQUA scores by the median. High c-Src expression was associated with decreased survival in primary specimens $(P=0.0027)$, but not in metastatic cases $(\mathrm{P}=0.17)$; Figure 1(D-E).

Given the association between melanoma progression and SFK expression, we assessed the effects of dasatinib on cell viability in eight melanoma cell lines and determined timedependent effects of dasatinib on its targets (Figure S2). $\mathrm{IC}_{50}$ s ranged from $100 \mathrm{nM}$ to 9.65 $\mu \mathrm{M}$ (Table S2). Clear differences were seen between the two most sensitive cell lines, YULAC and YUROB, and the others ( $\mathrm{IC}_{50} \mathrm{~s}<300 \mathrm{nM}$ and $\mathrm{IC}_{50} \mathrm{~s}>4 \mu \mathrm{M}$, respectively). YULAC had a B-Raf mutation, and YUROB was wild type. Both YULAC and YUROB did not harbor exon 11, 13, or $17 \mathrm{c}$-Kit mutations. C-Kit protein was detected by Western blot in YUMAC and MEL501 cell lines only, and neither had a c-Kit mutation. $\mathrm{IC}_{50}$ s for YUROB and YULAC were in the range of the serum concentration obtained in dasatinib phase I trials (Demetri et al., 2009), while $\mathrm{IC}_{50}$ s for the other cell lines were higher.

As with other targeted therapies, it is possible that response to Src targeting drugs is associated with expression levels of the target, downstream mediators or members of parallel pathways, and quantitative assays are needed to predict response. Given that in cells that do not harbor c-Kit mutations or BCR-ABL translocations, predictors of dasatinib response have not been identified, and dasatinib has a broad range of targets, we employed a relatively broad approach to identify predictors of response using reverse phase protein arrays (RPPAs). The RPPAs included a number of dasatinib targets, direct or indirect downstream Src effectors, and members of crosstalk pathways including MAPK, PI3K, and FAK pathways (Table S3). Levels of phospho-c-Src, total c-Src, and their direct downstream effectors - phosphorylated STAT3 and STAT5 - were not associated with dasatinib sensitivity.

The most differentially expressed protein by RPPA between sensitive and resistant cell lines was caveolin-1, which was high in YULAC and YUROB, Figure 2A. Although the number of cell lines was small, a significant association was found between high caveolin-1 levels and increased sensitivity (low $\mathrm{IC}_{50} ; \mathrm{P}=0.0004$ ). This is consistent with reports in breast and 
ovarian cancer using preclinical models (Huang et al., 2007; Konecny et al., 2009). We verified the RPPA results by Western blot (Figure 2B); caveolin-1 levels were high in YULAC and YUROB, compared to dasatinib-resistant cell lines.

Using AQUA, we assessed the associations between caveolin-1 expression and dasatinib activity in pretreatment tumors from 21 patients enrolled in a phase II trial of dasatinib. Our cohort was small, as detailed in the study by (Kluger et al., 2010), and included two patients with partial responses, four with tumor reduction that did not meet partial response criteria, and fifteen with no tumor reduction (Figure 2C and Figure S3). We dichotomized AQUA scores by the median and classified tumors as 'high' or 'low' expressers. By $\chi^{2}$-analysis on this small sample, we found a trend toward an association between elevated caveolin- 1 and response to therapy (Figure 2D). Five of six patients who had tumor reduction had caveolin-1 expression levels above the median ( $\mathrm{P}=0.06)$, suggesting that caveolin-1 might be a valuable predictive marker for dasatinib sensitivity, a finding that needs to be confirmed in other clinical trials. Caveolin-1 is a Src substrate that participates in the modulation of cSrc kinase activity and mediates the activation of Fyn by integrins, required for anchoragedependent cell growth (Wary et al., 1998). Our results further support the finding that dasatinib inhibits invasion and metastatic spread of cancer cells (Buettner et al., 2008).

In summary, our studies suggest that SFKs might be important therapeutic targets in melanoma and indicate that a subset of melanomas is dasatinib-sensitive. High caveolin-1 levels were seen in dasatinib-sensitive cell lines and in five of six tumors from metastatic melanoma patients treated with dasatinib who had tumor shrinkage. The number of patients treated on our phase II clinical trial was small, and the predictive value of caveolin-1 requires further confirmation in other (ongoing) clinical trials of dasatinib-containing regimens for melanoma.

\section{Supplementary Material}

Refer to Web version on PubMed Central for supplementary material.

\section{Acknowledgments}

This work was supported by National Institutes of Health grant [number: CA115756-01] (to H. Kluger), by the Yale SPORE in Skin Cancer, [number: 1 P50 CA121974-01] (to R. Halaban); and by the Milstein-Meyer Fund for Melanoma Research at Yale. The M. D. Anderson Cancer Center Reverse Phase Protein Array Core Facility is supported by National Cancer Institute - Cancer Center Support Grant [number: CA-16672].

\section{References}

Barnekow A, Paul E, Schartl M. Expression of the c-src protooncogene in human skin tumors. Cancer Res. 1987; 47:235-240. [PubMed: 2431764]

Buettner R, Mesa T, Vultur A, Lee F, Jove R. Inhibition of Src family kinases with dasatinib blocks migration and invasion of human melanoma cells. Mol Cancer Res. 2008; 6:1766-1774. [PubMed: 19010823]

Demetri GD, Lo Russo P, Macpherson IR, Wang D, Morgan JA, Brunton VG, Paliwal P, Agrawal S, Voi M, Evans TR. Phase I dose-escalation and pharmacokinetic study of dasatinib in patients with advanced solid tumors. Clin Cancer Res. 2009; 15:6232-6240. [PubMed: 19789325]

Huang J, Asawa T, Takato T, Sakai R. Cooperative roles of Fyn and cortactin in cell migration of metastatic murine melanoma. J Biol Chem. 2003; 278:48367-48376. [PubMed: 13129922] 
Huang F, Reeves K, Han X, Fairchild C, Platero S, Wong TW, Lee F, Shaw P, Clark E. Identification of candidate molecular markers predicting sensitivity in solid tumors to dasatinib: rationale for patient selection. Cancer Res. 2007; 67:2226-2238. [PubMed: 17332353]

Jilaveanu LB, Zito CR, Aziz SA, Conrad PJ, Schmitz JC, Sznol M, Camp RL, Rimm DL, Kluger HM. $\mathrm{C}$-Raf is associated with disease progression and cell proliferation in a subset of melanomas. Clin Cancer Res. 2009; 15:5704-5713. [PubMed: 19737955]

Kluger HM, Dudek AZ, Mccann C, Ritacco J, Southard N, Jilaveanu LB, Molinaro A, Sznol M. A phase 2 trial of dasatinib in advanced melanoma. Cancer. 201010.1002/cncr.25766

Konecny GE, Glas R, Dering J, et al. Activity of the multikinase inhibitor dasatinib against ovarian cancer cells. Br J Cancer. 2009; 101:1699-1708. [PubMed: 19861960]

Loganzo F Jr, Dosik JS, Zhao Y, Vidal MJ, Nanus DM, Sudol M, Albino AP. Elevated expression of protein tyrosine kinase c-Yes, but not c-Src, in human malignant melanoma. Oncogene. 1993; 8:2637-2644. [PubMed: 7690926]

Wary KK, Mariotti A, Zurzolo C, Giancotti FG. A requirement for caveolin-1 and associated kinase Fyn in integrin signaling and anchorage-dependent cell growth. Cell. 1998; 94:625-634. [PubMed: 9741627]

Wellbrock C, Schartl M. Activation of phosphatidylinositol 3-kinase by a complex of p59fyn and the receptor tyrosine kinase Xmrk is involved in malignant transformation of pigment cells. Eur J Biochem. 2000; 267:3513-3522. [PubMed: 10848967] 

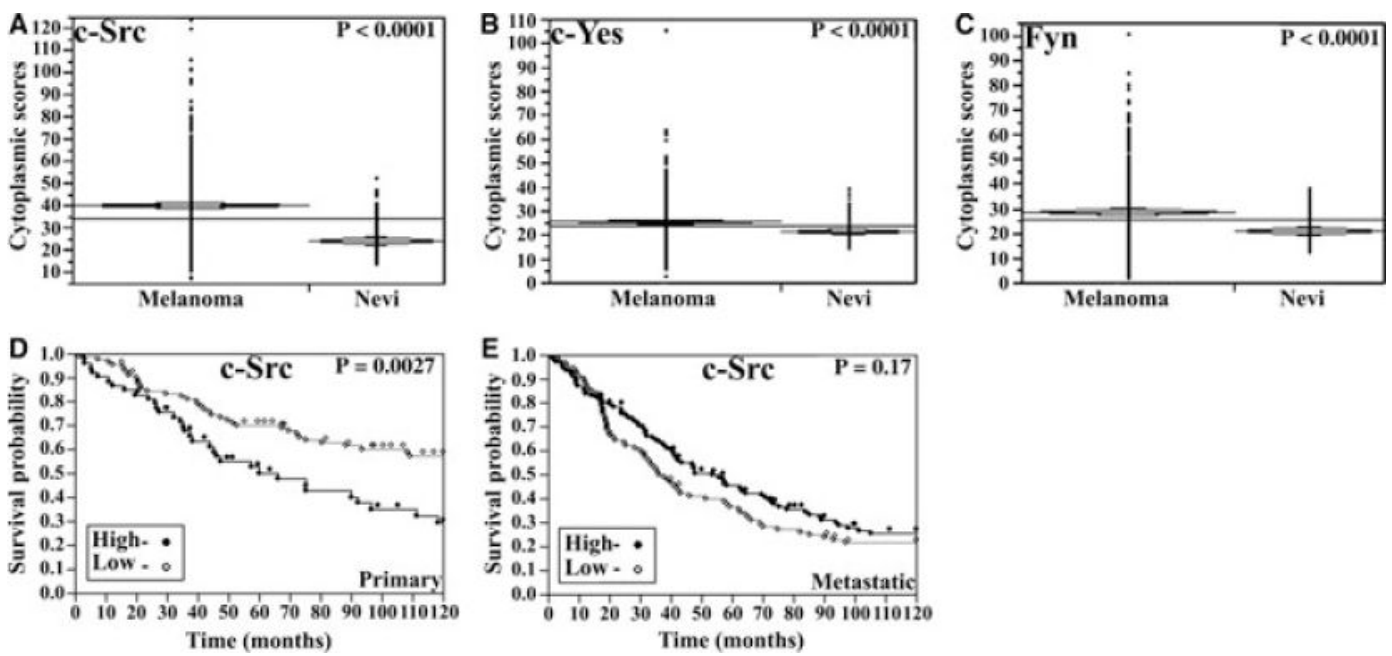

Figure 1.

Association between expression of Src proteins and tumor progression. (A-C) Means plots demonstrating differences in expression of c-Src, c-Yes and Fyn expression in nevi and malignant specimens (metastatic and primary melanomas). Horizontal lines reflect mean expression in the entire sample. By un-paired t-tests, expression of Src proteins was significantly higher in malignant versus benign tissue cores, although larger differences were seen for c-Src than for c-Yes and Fyn. (D, E) Kaplan-Meier survival curves for c-Src automated, quantitative analysis scores were divided by the median score for the primary and metastatic subsets of patients respectively. High c-Src expression is associated with poor survival in primary melanoma lesions but not in metastatic specimens. 
A
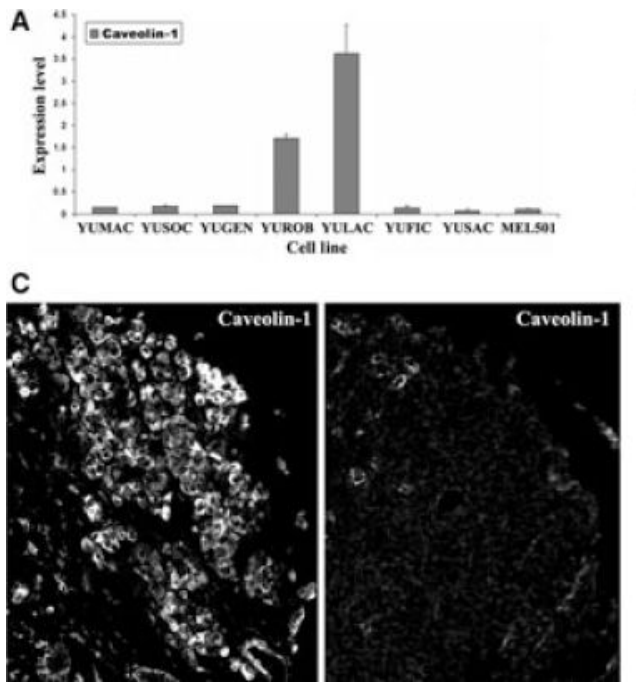

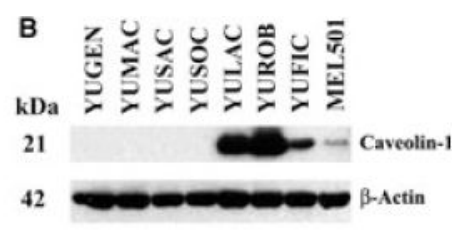

D

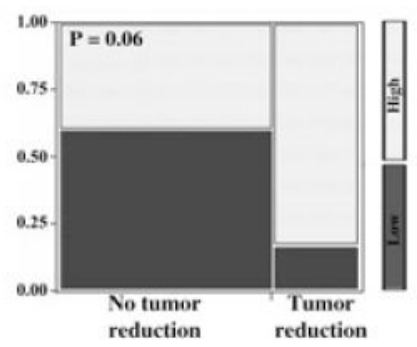

Figure 2.

Caveolin-1 expression in melanoma cell lines and pretreatment tumors of melanoma patients treated with dasatinib: Lysates were profiled by reverse phase protein array (A) and confirmed by Western blot analysis (B). Caveolin-1 was expressed at high levels in dasatinib sensitive compared to dasatinib-resistant cells. (C) Caveolin-1 expression was quantitatively assessed in pretreatment tumors of 21 patients who were enrolled in a phase II trial of dasatinib. Caveolin-1 staining was mostly membranous/cytoplasmic and automated, quantitative analysis scores ranged from 6.36 to 66.9 (median 13.4), with strong correlations between matching specimens from the same patient $(\mathrm{R}=0.89 ; \mathrm{P}<0.0001)$. Expression of caveolin- 1 at $40 \times$ is shown for two histospots of tumor samples derived from a patient who had a partial response (left panel) and a patient who had no response (right panel) to dasatinib. (D) Chi-squared $\left(\chi^{2}\right)$ analysis comparing high and low caveolin-1 expression in specimens of patients who did or did not have tumor reduction. We defined high caveolin-1 expression as expression that was equal to or higher than the median score (13.4). Five of six patients $(83.3 \%)$ who had either a partial response or tumor reduction with dasatinib treatment had high caveolin-1 expression, whereas 6 of 15 patients (40\%) who had no tumor reduction had high caveolin-1 levels $(\mathrm{P}=0.06)$. 